\title{
Estudio Cariométrico de Placentas de Ratones con Infección Aguda por Diferentes Cepas de Trypanosoma cruzi
}

\author{
Karyometric Study of Placentas from Mice with Acute Infection \\ by Different Strains of Trypanosoma cruzi

\begin{abstract}
"Miguel Angel Sala; ;,** Ruberval Armando Lopes; ***Ana Amélia Carraro Abrahão; ${ }^{* * * * *}$ Cícero Meneguette; ****érgio de
\end{abstract} \\ Albuquerque \& ${ }^{* * * * *}$ Rosa Domingues Ribeiro
}

SAlA, M. A.; LOPES, R. A.; ABRAHÃO, A. A. C.; MENEGUETTE, C.; ALBUQUERQUE, S. \& RIBEIRO, R. D. Estudio cariométrico de placentas de ratones con infección aguda por diferentes cepas de Trypanosoma cruzi. Int. J. Morphol., 26(3):623-627, 2008.

RESUMEN: El objetivo de este trabajo fue evaluar cariometricamente las alteraciones causadas por diferentes cepas de $T$. cruzi en la placenta del ratón. Ratones hembras de 60 días, grávidas, fueron inoculadas, intraperitonealmente, con $2 \times 10^{5}$ tripomastigotes sanguíneos de las cepas colombiana, Y, Bolivia o RC del T. cruzi. Fueron observadas claras diferencias en las alteraciones cariométricas de las células trofoblásticas gigantes y de las células trofoblásticas de la zona esponjosa. Los resultados demostraron que las cepas colombiana y RC causan alteraciones tanto en las células trofoblásticas gigantes como en las células del trofoblasto esponjoso, mientras que las cepas Y y Bolivia provocan alteraciones solamente en las células trofoblásticas gigantes. Es posible concluir que cada cepa posee características propias y que, a pesar del tipo similar de transmisión, presenta matices diferenciales en el proceso de la patogénesis placentaria.

PALABRAS CLAVE: Enfermedad de Chagas Experimental; Trypanosoma cruzi; Ratón; Placenta; Cariometría.

\section{INTRODUCCIÓN}

La enfermedad de Chagas, que afecta casi 20 millones de personas en Latinoamérica, puede ser adquirida a través de vectores, transfusión sanguínea, transplante de órganos, alimentos o leche materna contaminada o por transmisión transplacentaria.

Apenas dos años después del descubrimiento de la enfermedad, fue sugerida la posibilidad de la infección congénita (Chagas, 1911; Lozano et al., 1911). Luego de algunos intentos experimentales fracasados, Mayer \& RochaLima (1914), procurando obtener experimentalmente la transmisión congénita, detectaron nidos de amastigotes de Trypanosoma cruzi en placentas de ratón, aunque sin parasitismo fetal. En 1921, Nattan-Larrier obtuvo la transmisión vertical en fetos de cobayas, infectando ratones mediante la inoculación de sangre o líquido amniótico de esos fetos. Por otra parte, solamente en 1923, Villela demostró, sin lugar a dudas, la transmisión congénita en perros, detectando los tripanosomas en las placentas y fetos.
Según Nattan-Larrier (1928), la infección congénita ocurre a través de las comunicaciones entre la circulación materna y fetal, debido a lesiones placentarias causadas por el T. cruzi. Así, la lesión del trofoblasto permite que los tripomastigotes pasen de la sangre materna al estroma placentario, donde se multiplican y forman nidos de amastigotes en el interior de los macrófagos, que al romperse los liberan como tripomastigotes en la sangre fetal (Werner \& Kunert, 1958; Apt, 1965; Apt \& Diaz, 1965; Delgado \& Santos-Buch, 1978). Sin embargo, para otros autores, la transmisión congénita puede ocurrir sin necesidad de existir lesión previa del trofoblasto (Nattan-Larrier, 1921; Campos, 1928; Silva \& Nussenzweig, 1953; Mjihdi et al., 2002).

La enfermedad de Chagas presenta manifestaciones clínicas y patológicas variadas, provocando lesiones diversas en diferentes órganos. Se procuró explicar la heterogeneidad por la dualidad de formas sanguíneas del T. cruzi (Brener \& Chiari, 1963; 1965) y la variación del comporta-

* Departamento de Morfología, Estomatología y Fisiología, Facultad de Odontología de Ribeirão Preto, Universidad de São Paulo, Brasil.

** Universidad de Franca, UNIFRAN, Franca, São Paulo, Brasil.

**** Departamento de Ciencias de la Salud, Facultad de Ciencias Farmaceuticas de Ribeirão Preto, Universidad de São Paulo, Brasil.

***** Facultad de Medicina de São José do Rio Preto, SP, Brasil. 
miento de la infección según se originase de tripomastigotes o de amastigotes (Barreto et al., 1979). Otro factor de variación se debe a la diversidad de cepas. Estudios experimentales mostraron diferencias del patrón de transmisión congénita y de la severidad de la enfermedad entre diversas cepas de T. cruzi (Andrade et al., 1973: Andrade, 1982; Nunes et al., 1990). Aún hoy son poco conocidos los mecanismos de transmisión congénita, el parasitismo placentario y las lesiones causadas por las diferentes cepas en ese órgano.

El objetivo del presente trabajo es evaluar cariométricamente las posibles alteraciones placentárias en ratones infectados durante la gravidez con diferentes cepas de T. cruzi.

\section{MATERIAL Y MÉTODO}

Fueron usados 25 ratones blancos (Mus musculus), variedad Swiss, hembras vírgenes, de 60 días de edad. Después de dos semanas de adaptación, las hembras fueron colocadas para cruzar con machos fértiles durante la noche. La mañana siguiente, si comprobada la presencia de un tampón vaginal, ese día era considerado el primero de la preñez (DP1).

Las hembras preñadas, mantenidas en condiciones controladas de luz (12 h-luz, $12 \mathrm{~h}$-oscuridad), temperatura $\left(22^{\circ} \pm 2^{\circ} \mathrm{C}\right)$ y humedad $(55 \% \pm 10 \%)$, fueron alimentadas con ración comercial y agua ad limitum. Los animales fueron inoculados intraperitonealmente con $2 \times 10^{5}$ formas sanguíneas de tripomastigotes de diferentes cepas de T. cruzi, siendo constituidos, así, los siguientes grupos experimentales:

Grupo I - Inyectado el DP10 con suero fisiológico estéril (grupo control);

Grupo II - Inoculado el DP13 con la cepa colombiana del $T$. cruzi (Federici et al., 1964),

Grupo III - Inoculado el DP13 con la cepa Y del T. cruzi (Silva \& Nussenzweig, 1953),

Grupo IV - Inoculado el DP6 con la cepa Bolivia del T. Cruzi (Funayama \& Prado, 1974), y

Grupo V - Inoculado el DP8 con la cepa RC del T. cruzi (Albuquerque \& Barreto, 1968).

El día de inoculación fue programado de modo que los niveles máximos de parasitemia fuesen alcanzados al término del embarazo.

Todas las cepas de T. cruzi utilizadas son mantenidas en el Laboratorio de Parasitología de la Facultad de Ciencias Farmacéuticas de Ribeirão Preto, Universidad de São Paulo, Brasil.
Los animales fueron sacrificados en el DP19, por dislocación cervical bajo anestesia con tribromoetanol. Los fetos y anexos fueron retirados y rápidamente fijados en alcohol $80 \%$ - $85 \mathrm{~mL}$, formol $10 \mathrm{~mL}$ y ácido acético $-5 \mathrm{~mL}$, por $24 \mathrm{~h}$.

Luego de determinado el volumen (Scherle, 1970), cada placenta fue seccionada a lo largo del diámetro mayor, incluida en parafina, cortada con $6 \mu \mathrm{m}$ de espesor y teñida con hematoxilina y eosina.

El estudio cariométrico fue realizado en 50 núcleos de las células trofoblásticas gigantes y 50 núcleos del trofoblasto de la zona esponjosa, de cada placenta. Usando la metodología propuesta por Sala et al. (1994) fueron estimados los siguientes parámetros nucleares: diámetro medio, perímetro, área, superficie, volumen, relación superficie/volumen, relación entre diámetros, coeficiente de forma, índice de contorno y excentricidad.

Ya que la distribución de los datos, determinada mediante la prueba de Shapiro-Wilks, no fue normal para la mayoría de las variables estimadas, la comparación de los efectos causados por las diferentes cepas de T. cruzi sobre los núcleos de las células placentarias fue realizada con la prueba no paramétrica de Kruskal-Wallis (Sprent \& Smeeton, 2001).

\section{RESULTADOS}

Los parámetros nucleares de las células trofoblásticas gigantes de las placentas en los diferentes grupos considerados son vistos en la Tabla I. Puede ser observado que, cuando comparados a los controles, los animales infectados mostraron disminución del diámetro medio, área, superficie, volumen y coeficiente de forma, y aumento de la relación superficie / volumen, relación entre diámetros, índice de contorno y excentricidad. De este modo, los núcleos de las células trofoblásticas gigantes son menores y presentan formas más alargadas en los animales infectados.

La comparación de las variables de los núcleos de las células trofoblásticas gigantes de los grupos infectados por el T. cruzi, muestra que ocurrió mayor reducción del diámetro y perímetro nucleares en los grupos inoculados con las cepas Bolivia y RC, mientras que área, superficie y volumen fueron menores en los grupos infectados por la cepa RC. Las variables que evalúan la forma (relación entre diámetros, excentricidad, índice de contorno y coeficiente de forma) muestran que los núcleos de las células trofoblásticas gigantes fueron más alargados en las placentas de los animales inoculados con las cepas colombiana, Bolivia y RC. 
La Tabla II muestra los parámetros nucleares de las células del trofoblasto en la zona esponjosa de las placentas de los diferentes grupos. Se observa que en los grupos infectados por la cepa colombiana o RC hubo un aumento significativo del diámetro, perímetro, área, superficie y volu- men nuclear, mayor que en los otros grupos.

La forma de los núcleos de las células del trofoblasto de la zona esponjosa, en general, no mostró alteraciones significativas en los animales de los grupos infectados por el T. cruzi.

Tabla I. Parámetros nucleares de las células trofoblásticas gigantes de las placentas de los grupos control e inoculados con T. cruzi. Los resultados son presentados en forma de media \pm desvío estandar. Prueba de Kruskal-Wallis.

\begin{tabular}{|c|c|c|c|c|c|c|}
\hline \multirow[t]{2}{*}{ VARIABLE } & \multirow[b]{2}{*}{ Control } & \multirow[b]{2}{*}{ Colombiana } & \multicolumn{2}{|c|}{ CEPA } & \multirow[b]{2}{*}{$\mathbf{R C}$} & \multirow{2}{*}{$\begin{array}{l}\text { Kruskal- } \\
\text { Wallis }\end{array}$} \\
\hline & & & $\mathbf{Y}$ & Bolivia & & \\
\hline Diáme tro medio $(\mu \mathrm{m})$ & $18,8 \pm 0,2$ & $15,3 \pm 0.2^{\mathrm{a}}$ & $15,4 \pm 0,2^{\mathrm{a}}$ & $14,0 \pm 0,3^{\mathrm{b}}$ & $13,7 \pm 0,2^{\mathrm{b}}$ & $21,562 *$ \\
\hline Perímetro $(\mu \mathrm{m})$ & $60,4 \pm 0,7$ & $50,2 \pm 0,7^{\mathrm{a}}$ & $50,2 \pm 0,8^{\mathrm{a}}$ & $45,7 \pm 0.8^{\mathrm{b}}$ & $44,9 \pm 0,7^{b}$ & $21,227 *$ \\
\hline Área $\left(\mu \mathrm{m}^{2}\right)$ & $279,9 \pm 5,8$ & $183,2 \pm 5,6^{\mathrm{a}}$ & $187,0 \pm 5,7^{\mathrm{a}}$ & $152,9 \pm 6,7$ & $147,6 \pm 4,4$ & $21,588 *$ \\
\hline Superficie $\left(\mu \mathrm{m}^{2}\right)$ & $1119,6 \pm 23,2$ & $732,6 \pm 22,2^{\mathrm{a}}$ & $747,9 \pm 22,9^{a}$ & $611,6 \pm 22,6$ & $590,3 \pm 17,6$ & $21,585^{*}$ \\
\hline Volumen $\left(\mu \mathrm{m}^{3}\right)$ & $3522,6 \pm 109,5$ & $1864,5 \pm 84,8^{\mathrm{a}}$ & $1923,2 \pm 88,5^{\mathrm{a}}$ & $1422,1 \pm 78,9$ & $1348,6 \pm 60,5$ & $21,585^{*}$ \\
\hline Relación sup./Volumen & $0,32 \pm 0,01$ & $0,39 \pm 0,01^{\mathrm{a}}$ & $0,39 \pm 0,01^{\mathrm{a}}$ & $0,43 \pm 0,01^{\mathrm{b}}$ & $0,44 \pm 0,01^{\mathrm{b}}$ & $22,237 *$ \\
\hline Relación entre diámetros & $1,36 \pm 0,03$ & $1,65 \pm 0,05^{\mathrm{a}}$ & $1,55 \pm 0,03$ & $1,61 \pm 0,05^{\mathrm{a}}$ & $1,61 \pm 0,04^{\mathrm{a}}$ & $17,334^{*}$ \\
\hline Excentricidad & $0,68 \pm 0,02$ & $0,79 \pm 0,02^{\mathrm{a}}$ & $0,76 \pm 0,01$ & $0,78 \pm 0,02^{\mathrm{a}}$ & $0,78 \pm 0,01^{\mathrm{a}}$ & $16,652^{*}$ \\
\hline Coeficiente de forma & $0,97 \pm 0,01$ & $0,91 \pm 0,01^{\mathrm{a}}$ & $0,93 \pm 0,01$ & $0,92 \pm 0,01^{\mathrm{a}}$ & $0,92 \pm 0,01^{a}$ & $17,221 *$ \\
\hline Índice de contorno & $3,61 \pm 0,01$ & $3,71 \pm 0,02^{\mathrm{a}}$ & $3,67 \pm 0,01$ & $3,70 \pm 0,02^{\mathrm{a}}$ & $3,70 \pm 0,01^{\mathrm{a}}$ & $17,087 *$ \\
\hline
\end{tabular}

Letras iguales señalan ausencia de diferencias significativas $* \mathrm{p}<0,01$

Tabla II. Parámetros nucleares de las células del trofoblasto de la zona esponjosa de las placentas de los grupos control e inoculados con T. cruzi. Los resultados son presentados en forma de media \pm desvío estandar. Prueba de Kruskal-Wallis.

\begin{tabular}{|c|c|c|c|c|c|c|}
\hline \multirow[t]{2}{*}{ VARIABLE } & \multicolumn{5}{|c|}{ CEPA } & \multirow{2}{*}{$\begin{array}{c}\text { Kruskal- } \\
\text { Wallis }\end{array}$} \\
\hline & Control & Colombiana & $\mathbf{Y}$ & Bolivia & $\mathbf{R C}$ & \\
\hline Diámetro medio $(\mu \mathrm{m})$ & $6,2 \pm 0,3$ & $7,4 \pm 0,3^{\mathrm{a}}$ & $5,9 \pm 0,1^{\mathrm{b}}$ & $5,8 \pm 0,2^{b}$ & $6,8 \pm 0,3^{\mathrm{a}}$ & $19,560 * *$ \\
\hline Perímetro $(\mu \mathrm{m})$ & $19,9 \pm 0,9^{\mathrm{a}}$ & $23,6 \pm 1,1^{b}$ & $19,0 \pm 0,2^{\mathrm{a}, \mathrm{c}}$ & $18,6 \pm 0,7^{\mathrm{c}}$ & $21,6 \pm 0,8^{b}$ & $19,571 * *$ \\
\hline Área $\left(\mu \mathrm{m}^{2}\right)$ & $31,1 \pm 2,9^{\mathrm{a}}$ & $43,6 \pm 3,6^{\mathrm{b}}$ & $28,0 \pm 0,5^{\mathrm{a}, \mathrm{c}}$ & $27,2 \pm 2,2^{\mathrm{c}}$ & $36,8 \pm 2,9^{b}$ & $19,379 * *$ \\
\hline Superficie $\left(\mu \mathrm{m}^{2}\right)$ & $124,3 \pm 11,8^{\mathrm{a}}$ & $174,4 \pm 14,3$ & $112,1 \pm 2,2^{\mathrm{a}, \mathrm{b}}$ & $108,8 \pm 8,7^{\mathrm{b}}$ & $141,2 \pm 6,4$ & $19,505^{* *}$ \\
\hline Volumen $\left(\mu \mathrm{m}^{3}\right)$ & $132,6 \pm 19,1^{\mathrm{a}}$ & $223,8 \pm 26,7^{\mathrm{b}}$ & $114,4 \pm 3,9^{\mathrm{a}}$ & $109,6 \pm 13,3^{\mathrm{a}}$ & $172,0 \pm 21,26^{b}$ & $18,903 * *$ \\
\hline Relación sup./Volumen & $0,94 \pm 0,05^{\mathrm{a}}$ & $0,78 \pm 0,03^{\mathrm{b}}$ & $0,98 \pm 0,01^{\mathrm{a}}$ & $1,00 \pm 0,04^{\mathrm{a}}$ & $0,86 \pm 0,04^{\mathrm{b}}$ & $18,740^{* *}$ \\
\hline Relación entre diámetros & $1,26 \pm 0,05$ & $1,32 \pm 0,09$ & $1,32 \pm 0,05$ & $1,26 \pm 0,04$ & $1,22 \pm 0,04$ & 8,597 \\
\hline Excen tricidad & $0,52 \pm 0,06$ & $0,53 \pm 0,06$ & $0,54 \pm 0,05$ & $0,49 \pm 0,04$ & $0,45 \pm 0,06$ & 5.788 \\
\hline Coeficiente de forma & $0,97 \pm 0,01$ & $0,96 \pm 0,01$ & $0,96 \pm 0,01$ & $0,97 \pm 0,01$ & $0,97 \pm 0,01$ & 8,763 \\
\hline Índice de contorno & $3,59 \pm 0,01^{\mathrm{a}, \mathrm{b}}$ & $3,61 \pm 0,01^{\mathrm{a}, \mathrm{c}}$ & $3,61 \pm 0,01^{\mathrm{c}}$ & $3,59 \pm 0,01^{\mathrm{a}, \mathrm{c}, \mathrm{d}}$ & $3,58 \pm 0,01^{\mathrm{b}, \mathrm{d}}$ & $9,560 *$ \\
\hline
\end{tabular}

Letras iguales señalan ausencia de diferencias significativas $* \mathrm{p}<0,05 * * \mathrm{p}<0,01$

\section{DISCUSIÓN}

Varios autores señalaron diferencias en la distribución, intensidad y tipo de infiltrado inflamatorio, así como en el tipo de lesión provocada por diferentes cepas de $T$. cruzi en los tejidos de los animales infectados (Andrade, 2000). Quizás las diferencias debidas a la cepa del parásito sean capaces de alterar las manifestaciones de la enferme- dad. Brener (1969) estableció una relación entre el tipo morfológico de las formas sanguíneas de T. cruzi y la susceptibilidad a los anticuerpos. Así, las formas delgadas tendrían mayor capacidad de invasión tisular y mayor susceptibilidad a los anticuerpos. El poder patógeno de la cepa se relaciona con su capacidad de lesionar los tejidos, mientras 
que la virulencia resulta de su capacidad de multiplicación que, a pesar de variar dentro de ciertos límites, es característica de la cepa (Andrade, 2000). La acción concomitante de virulencia y poder patógeno puede determinar el curso de la infección.

El presente trabajo mostró claras diferencias en las alteraciones nucleares de las células trofoblásticas gigantes y de las células del trofoblasto de la zona esponjosa causadas por la infección experimental por diversas cepas de T. cruzi.

De este modo, las cepas Y y Bolivia provocaron disminución del volumen del núcleo de las células trofoblásticas gigantes, mientras que la cepa Y fue la que causó menor alteración de la forma nuclear. Ambas cepas no causaron alteraciones de tamaño o de forma en los núcleos de las células trofoblásticas de la zona esponjosa.

Las cepas colombiana y RC provocaron aumento significativo del tamaño nuclear en el trofoblasto de la zona esponjosa y disminución del volumen nuclear en las células trofoblásticas gigantes. Ambas cepas provocaron alteraciones en la forma nuclear de las células trofoblásticas gigantes, siendo más marcadas con la cepa colombiana.

Los resultados permiten inferir que cada cepa posee características propias y que, a pesar del tipo semejante de transmisión, presentan matices diferenciales en el proceso patogénico. Así, las cepas Bolivia y RC que causan alteraciones bastante similares en las células trofoblásticas gigantes, muestran mayor histotropismo por esas células, con presencia de nidos de amastigotes dentro de las mismas (observaciones no publicadas), confirmando los resultados de Delgado \& Santos-Buch (1978) y Andrade (1982). De este modo, la transmisión congénita dependería de la cepa y de la capacidad de fagocitosis de los macrófagos placentarios. Así, la virulencia y capacidad patógena de las cepas tienen papel importante en la transmisión congénita, independientemente de su origen (Delgado \& Santos-Buch, 1978; Andrade, 1982; Macedo \& Pena, 1990; Moreno et al., 2003).

SAlA, M.A.; LOPES, R.A.; ABRAHÃO, A.A.C.; MENEGUETTE, C.; ALBUQUERQUE, S. \& RIBEIRO, R.D. Karyometric study of placentas from mice with acute infection by different strains of Trypanosoma cruzi. Int. J. Morphol., 26(3):623-627, 2008.

SUMMARY: The objective of this work was to evaluate karyometrically the alterations caused by different strains of Trypanosoma cruzi in the mouse placenta. Pregnant mice, 60-day old, were intraperitoneally inoculated with $2 \times 10^{5}$ bloodstream trypomastigotes of the Colombian, Y, Bolivia or RC strain of $T$. cruzi. There were observed clear differences in the karyometric alterations of the trophoblast giant cells and in the spongiotrophoblast cells. The results demonstrate that the Colombian and RC strains cause alterations both in the trophoblast giant cells and in the spongiotrophoblast cells, whereas the Y and Bolivia strains provoke alterations only in the trophoblast giant cells. It is possible concluding that each strain has its own characteristics and that, in spite of the similar type of transmission, it show differential nuances in the placental pathogenic process.

KEY WORDS: Experimental Chagas' disease; Trypanosoma cruzi; Mouse; Placenta; Karyometry.

\section{REFERENCIAS BIBLIOGRÁFICAS}

Albuquerque, R. D. R. \& Barreto, M. P. Estudos sobre reservatórios e vetores silvestres do trypanosoma cruzi. XXX - Infecção natural do cachorro-do-mato, Cerdocyon thous azarae (Wied, 1824) pelo T. cruzi. Rev. Bras. Biol., 28:457-68, 1968.

Andrade, S. G. The influence of the strain of Trypanosoma cruzi in placental infections in mice. Trans. Royal Soc. Trop. Méd. Hyg., 76:123-8, 1982.

Andrade, S. G. Patologia experimental da doença de Chagas. In: Brener, Z.; Andrade, Z. A. \& Barral-Netto, M. (eds.). Trypanosoma cruzi e Doença de Chagas. $2^{\mathrm{a}}$ ed. Rio de Janeiro, Guanabara Koogan, 2000. pp. 177-200.
Andrade, S. G.; Bittencourt, A. C. L.; Figueira, R. M. \& Carvalho, M. L. Estudo experimental de amostras do Trypanosoma cruzi isoladas de gestantes chagásicas. Rev. Patol. Trop., 2: 301-10, 1973.

Apt, W. \& Diaz, M. Hallazgo de Trypanosoma cruzi en estroma de las vellosidades coriales de ratones. Bol. Chil. Parasit., 20:52-3, 1965.

Apt, W. Transmisión congénita del Trypanosoma cruzi. I. Efecto de la thalidomide sobre la infección de los ratones por $T$. cruzi. Biológica (Santiago), 37: 28-44, 1965.

Barreto, M. P.; Ribeiro, R. D. \& Belda Neto, F. M. Estudos 
sobre reservatórios e vetores silvestres do Trypanosoma cruzi. LXXIV. Comportamento de amastigotas e tripomastigotas de cultura de amostras diversas do T. cruzi incubados com soro humano normal e inoculados em camundongos. Rev. Bras. Biol., 39:897-9, 1979.

Brener, Z. The behavior of slender and stout forms of Trypanosoma cruzi in the blood-stream of normal and immune mice. Ann. Trop. Med. Parasitol., 63:215-20, 1969.

Brener, Z. \& Chiari, E. Variações morfológicas observadas em diversas amostras do T. cruzi. Rev. Inst. Med. Trop., 5:2204, 1963.

Brener, Z. \& Chiari, E. Aspects of early growth of different Trypanosoma cruzi strain in culture medium. J. Parasitol., 51:922-6, 1965.

Campos, E. S. Transmissão intra-uterina do Trypanosoma cruzi na infecção experimental do cão. An. Fac. Med. São Paulo, 3:35-9, 1928.

Chagas, C. Nova entidade mórbida do homem. Resumo geral dos estudos etiológicos e clínicos. Mem. Inst. Oswaldo Cruz, 3:219-75, 1911.

Delgado, M. A. \& Santos-Buch, C. A. Transplacental transmission and fetal parasitosis of Trypanosoma cruzi in outbred white Swiss mice. Am. J. Trop. Med. Hyg., 27:110815, 1978.

Federici, E. E.; Abelmann, W. B. \& Neva, F. A. Chronic and progressive myocarditis and myositis in $\mathrm{C} 3 \mathrm{H}$ mice infected with Trypanosoma cruzi. Am. J. Trop. Med. Hyg., 113:27280, 1964.

Funayama, G. K. \& Prado, J. C. Jr. Estudo dos caracteres de uma amostra boliviana do T. cruzi. Rev. Inst. Med. Trop., 8:75-81, 1974.

Lozano, N.; Maggio, C. \& Rosenbuch, F. La enfermedad de Chagas. Arch. Hig., 4: 123-76, 1911.

Macedo, A. M. \& Pena, S. D. J. Genetic variability of Trypanosoma cruzi: implications for the pathogenesis of Chagas' disease. Parasitol. Today, 14:119-24, 1998.

Mayer, M. \& Rocha-Lima, H. Zum vernhalten von Schizotrypanum cruzi in Warmtblutern und Arthropoden. Arch. Schiffs Tropenhyg., 18:101-36, 1914.

Mjihdi, A.; Lambot, M. A.; Stewart, I. J.; Detournay, O.; Noel, J. C.; Carlier, Y. \& Truyens, C. Acute Trypanosoma cruzi infection in mouse induces infertility or placental parasite invasion and ischemic necrosis associated with massive fetal loss. Am. J. Pathol., 161:673-80, 2002.

Moreno, E. A.; Rivera, I. M.; Moreno, S. C.; Alarcón, M. E. \& Lugo-Yarbuh, A. Transmisión vertical de Trypanosoma cruzi en ratas Wistar durante la fase aguda de la infección. Invest. Clin., 44:241-54, 2003.

Nattan-Larrier, L. A. A. Heredité des infections experimentales a Schizotrypanum cruzi. Bull. Soc. Pathol. Exot., 14:232-8, 1921.

Nattan-Larrier, L. A. A. L'heredité de la maladie de Chagas. Bull. Acad. Nac. Med. (Paris), 99:97-100, 1928.

Nunes, M. P.; Coutinho, S. G.; Louis, J. A. \& Souza, W. J. Trypanosoma cruzi: quantification in tissue of experimentally infected mice by limiting dilution analysis. Exp. Parasitol., 70:186-92, 1990.

Sala, M. A.; Komesu, M. C.; Lopes, R. A. \& Maia Campos, G. Karyometric study of basal cell carcinoma. Braz. Dent. J., 5:11-4, 1994.

Scherle, W. A simple method for volumetry of organs in quantitative stereology. Mikroskopie, 26:57, 1970.

Silva, L. H. P. \& Nussenzweig, V. Sobre uma cepa de Trypanosoma cruzi altamente virulenta para o camundongo branco. Folia Clin. Biol., 20:191-203, 1953.

Sprent, P. \& Smeeton, N. C. Applied nonparametric statistical methods. $3^{\text {rd }}$ ed. Boca Raton: Chapman \& Hall/CRC, 2001.

Villela, E. A. A transmissão intra-uterina da moléstia de Chagas. Encefalite congênita pelo Trypanosoma cruzi (Nota prévia). Folia Méd., 4:41-3, 1923.

Werner, H. \& Kunert, H. Über die Ursache von congenitalen Protozon-Infektionen. Z. Tropenmed. Parasitol., 9: 17-27, 1958.

Dirección para correspondencia:

Prof. Dr. Miguel Angel Sala

Departamento de Morfologia, Estomatologia e Fisiologia

Faculdade de Odontologia de Ribeirão Preto

Universidad de Såo Paulo

Avenida do Café s/n

CEP 14040-904

Ribeirão Preto - SP

BRASIL

Email: masdmatt@forp.usp.br

Recibido : 15-11-2007 Aceptado: 15-09-2008 
